\title{
Booster medication to achieve capsule excretion in colon capsule endoscopy: a randomized controlled trial of three regimens
}

(1) $\odot \ominus$

\author{
Authors \\ Rasmus Kroijer ${ }^{1,2}$, Anne-Kirstine Dyrvig1,3, Morten Kobaek-Larsen ${ }^{1,2}$, Jens Overgaard Støvring ${ }^{4}$, Niels Qvist ${ }^{1,2}$, \\ Gunnar Baatrup ${ }^{1,2}$
}

Institutions

1 Department of Surgery, Odense University Hospital, Odense, Denmark

2 Department of Clinical Science, University of Southern Denmark, Denmark

3 Odense Patient Data Explorative Network OPEN, University of Southern Denmark, Odense, Denmark

4 Department of Surgery, Hospital South West Jutland, Esbjerg, Denmark

submitted 11.4.2018

accepted after revision 31.7.2018

Bibliography

DOI https://doi.org/10.1055/a-0732-494 |

Endoscopy International Open 2018; 06: E1363-E1368

(c) Georg Thieme Verlag KG Stuttgart · New York

ISSN 2364-3722

Corresponding author

Rasmus Kroeijer, Odense University Hospital - Department of Surgery, Baagøes alle 15 Svendborg 5700, Denmark Rasmus.Kroeijer@rsyd.dk

\section{ABSTRACT}

Background and study aims To achieve a complete colon capsule endoscopy, the entire colon must be visualized, clean and filled with clear fluids. The primary aim was to compare three booster regimens in colon capsule endos- copy in achieving capsule excretion within recording time. Secondary aims were quality of bowel cleansing and completion rate (both adequate cleansing and capsule excretion).

Patients and methods Patients scheduled for follow-up colonoscopy due to previous neoplastic findings or familial history of colorectal cancer aged 18 to 70 years were eligible. Bowel preparation was 2-L split doses of polyethylene glycol. Patients were randomized to three booster regimens of either polyethylene glycol (Group A), sulfate-based solution (Group B) or polyethylene glycol with iodine oral contrast (Group C).

Results One hundred eighty participants were included and randomized into three groups of 60 . Capsule excretion was $70 \%(95 \% \mathrm{Cl}: 58-80)$ in Group A, $73 \%(95 \% \mathrm{Cl}: 61-83)$ in Group B and in $68 \%$ (95\% Cl: $56-79)$ in Group C, no statistically significant differences. Bowel cleansing grade was statistically significant better in Group B compared to Group A $(P=0.03)$, but there were no statistically significant differences between Groups $C$ and $A(P=0.40)$. Complete examination rate was $65 \%(95 \% \mathrm{Cl}: 53-77), 72 \%(95 \% \mathrm{Cl}$ : $61-83)$ and $62 \%(95 \% \mathrm{Cl}: 50-74)$ in Group A, B and C respectively, not statistically significant different.

Conclusions Sulfate-based solution resulted in statistically significant better bowel cleansing compared to polyethylene glycol. Overall the excretion and completion rate was suboptimal. Achieving a high completion rate using patient-tolerable and low-risk compounds is still a challenge.

\section{Introduction}

Colon capsule endoscopy (CCE) is an emerging method for detection of colorectal pathology. Polyp detection rate and polyp sensitivity have been reported to be comparable to or better than conventional optical colonoscopy (OC) [1 - 3]. To achieve a complete investigation of the colon and rectum for diagnostic purposes, the bowel must be clean, containing clear fluids and the capsule must reach the anal verge within recording time. An overview of the basic principles in colon capsule endoscopy preparation is shown in - Fig. $\mathbf{1}$.

Bowel preparation is often administered as a split dose of $4-\mathrm{L}$ iso-osmotic polyethylene-glycol solution (PEG), but may be poorly tolerated by patients due to bad taste and large volume. One randomized controlled trial found that the effect of a $2-\mathrm{L}$ split-dose PEG was similar to the 4-L split in respect to cleansing quality and capsule excretion [4]. 


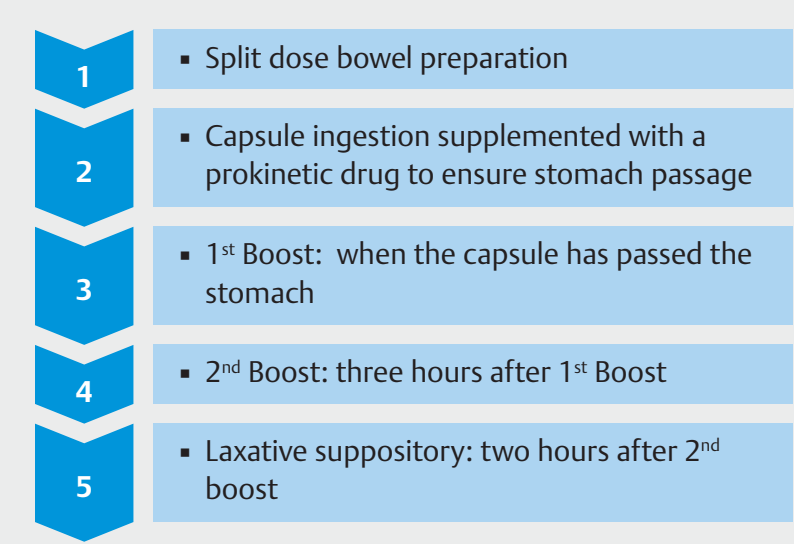

- Fig. 1 Overview of principal steps in colon capsule endoscopy preparation and examination.

Accordingly, we conducted a trial on 253 individuals undergoing colorectal cancer screening using a low-volume, low-risk regimen of 2-L split-dose PEG for preparation and PEG for boosters as well. The excretion rate was $57 \%$ [1] which illustrates the challenge of achieving both adequate preparation and timely propulsion of the capsule without a more powerful booster. The boosters commonly used in published trials are sodium phosphate, magnesium citrate, PEG, iodine oral contrast solution and sulfate-based solutions, with reported adequate cleansing rates from $61 \%$ to $95 \%$ and excretion rates from $64 \%$ to $97 \%$ [3-18].

The primary aim of this study was to compare the effect of three CCE booster regimens in achieving capsule excretion in an out-patient setting. Secondary aims were adequate cleansing rate, completion rate (both adequate cleansing and excretion), colon transit time, patient tolerability and compliance.

\section{Patients and methods}

The trial was a randomized controlled trial with one-sided investigator blinding, monitored by the regional good clinical practice unit, and reported according to the CONSORT statement [19]. Patients scheduled for follow-up colonoscopy at Odense University Hospital and Hospital of Southwest Jutland between February 1st, 2017 and November 1st, 2017 were screened. Inclusion criteria were follow-up due to previous neoplastic findings or familial history of colorectal cancer and age 18 to 70 years. Exclusion criteria were previous bowel surgery except appendectomy, renal insufficiency, pacemaker, pregnancy, breastfeeding, inflammatory bowel disease or allergies towards active substances administered in the trial. All participants who commenced bowel preparation were included in the analyses as intention to treat.

Participants were randomized in a 1:1:1 ratio to three different booster regimens. Randomization was stratified for the two centers ensuring an equal number of participants in the three study arms at each center. The regional pharmacy at Odense University Hospital prepackaged 60 medication regimens for each group, randomized the sequence and labelled each box with a randomization number. These numbers were consecutively assigned to patients. Investigators performing assessment of outcomes and data analyses were blinded to randomization until data acquisition was finished and the database was locked. Participants and the staff delivering the capsules and boosters were not blinded to randomization due to the different administration and preparation of the booster solutions.

Bowel preparation occurred at home. All participants received a bowel preparation consisting of magnesium tablets, $2-\mathrm{L}$ split-dose PEG solution (Moviprep, Norgine, Denmark) and were kept on a diet of watery fluids. Capsule delivery, unpackaging and instruction of booster medication were done in an outpatient clinic at the two centers. The booster regimens were: Group A: PEG solution (Moviprep, Norgine, Denmark); Group B: Sulfate-based solution (Eziclen, Ipsen limited, United Kingdom); Group C: PEG solution (Moviprep, Norgine, Denmark) and iodine oral contrast solution (Gastrografin, Bayer Group, Germany).

Exact dosage and timing of bowel preparation, capsule ingestion, prokinetic drug and boosters are shown in > Fig. 2 . Participants were instructed both orally and in writing on how to comply with bowel preparation and booster regimens and could phone a study nurse at any time during preparation and investigation. The capsules (Pillcam Colon 2, Medtronic, United States) were delivered by trained staff (Corporate Health, Odense, Denmark). Participants returned the belt recorder the day after capsule ingestion and completed a questionnaire rating their compliance with $(<25 \%, 25-75 \%$ or $>75 \%)$ and tolerability of $(0-100)$ the booster solutions. Patients completed the questionnaire electronically at the outpatient clinic. The CCE videos were uploaded to a diagnostic center (Corporate Health, Hamburg, Germany) which completed investigation reports using a dedicated software (Rapid Reader 7.0, Medtronic, United States). Reports included time of capsule ingestion, first cecal and last rectal image time (if available) and image, size and location of all polyp findings. Bowel cleanliness was graded according to the validated Leighton-Rex scale from 1-4 (1: Poor, 2: Fair, 3: Good, 4: Excellent) [20]. Bowel cleansing grade 2 to 4 was regarded as adequate for clinical purposes. An investigation with no images of the colon due to slow transit was regarded as bowel cleansing grade 1 . A video with images of the anal verge was regarded as excreted.

\section{Sample size and statistics}

The trial was performed as a randomized "pick a winner" intention-to-treat design, given the assumption that the best treatment would have an excretion rate of $90 \%$ as reported by Togashi et al. [11], with a margin of at least $15 \%$ to the runner-up treatment, and that the worst treatment achieving an excretion rate of $60 \%$ as reported by Kobaek-Larsen et al. [1]. Forty-nine participants in each arm would imply a minimum of $90 \%$ probability that the treatment with the highest excretion rate in the study is true. Assuming a $10 \%$ drop-out rate, the accrual was 56 participants in each arm or a total of 168 participants. The sample size was set at 180 participants, with 60 participants in each 


\begin{tabular}{|c|c|c|c|}
\hline \multicolumn{4}{|l|}{ Bowel preparation } \\
\hline Day - 2 & \multicolumn{3}{|c|}{$\begin{array}{l}\text { Two magnesia tablets in the morning } \\
\text { Two magnesia tablets in the evening } \\
2 \mathrm{~L} \text { of water }\end{array}$} \\
\hline Day - 1 & \multicolumn{3}{|l|}{$\begin{array}{l}\text { Clear liquids diet } \\
1 \mathrm{~L} \text { Moviprep }{ }^{\circledast} \text { solution } \\
2.5 \text { L water }\end{array}$} \\
\hline Day 0 & \multicolumn{3}{|l|}{$\begin{array}{l}\text { Clear liquids diet } \\
1 \mathrm{~L} \text { Moviprep }{ }^{\circledast} \text { solution } \\
1.5 \mathrm{~L} \text { water } \\
60-90 \text { minutes of fasting } \\
\text { Capsule ingestion } \\
20 \text { mg Domperidon }\end{array}$} \\
\hline Booster regimen & Group A & Group B & Group C \\
\hline $\begin{array}{l}\text { Signal } 1 \\
\text { (capsule reached the small bowel) }\end{array}$ & $\begin{array}{l}0.75 \mathrm{~L}_{\text {Moviprep }}{ }^{\circledR} \text { solution } \\
0.75 \mathrm{~L} \text { water }\end{array}$ & $\begin{array}{l}0.25 \text { L Eziclen }^{\circledR} \text { solution } \\
0.75 \text { L water }\end{array}$ & $\begin{array}{l}0.75 \mathrm{~L}_{\text {Moviprep }}{ }^{\circledR} \text { solution } \\
50 \mathrm{~mL} \text { Gastrografin } \\
0.75 \mathrm{~L} \text { water }\end{array}$ \\
\hline $\begin{array}{l}\text { Signal } 2 \\
\text { ( } 3 \text { hours after signal } 1 \text { ) }\end{array}$ & $\begin{array}{l}0.25 \mathrm{~L}_{\text {Moviprep }}{ }^{\circledR} \text { solution } \\
0.25 \mathrm{~L} \text { water }\end{array}$ & $\begin{array}{l}0.25 \text { L Eziclen }^{\circledast} \text { solution } \\
0.75 \mathrm{~L}_{\text {water }}\end{array}$ & $\begin{array}{l}0.25 \text { L Moviprep }^{\circledR} \text { solution } \\
25 \mathrm{~mL} \text { Gastrografin } \\
0.25 \text { L water }\end{array}$ \\
\hline $\begin{array}{l}\text { Signal } 3 \\
\text { ( } 2 \text { hours after signal } 2 \text { ) }\end{array}$ & \multicolumn{3}{|c|}{$\begin{array}{l}10 \text { mg rectal Bisacodyl } \\
1 \text { hour after signal 3: no dietary restrictions }\end{array}$} \\
\hline
\end{tabular}

- Fig. 2 Dosage and timing of bowel preparation and boosters.

group. In all statistical analyses patients were analyzed as one group regardless of center of inclusion.

Differences in proportions between groups were compared using chi squared test and $95 \%$ confidence intervals. Difference in bowel cleansing grade was estimated using Kruskal-Wallis linear regression and Dunn's multiple comparisons test, with group A as control group. When comparing the three groups, only in case of overall statistically significant difference among groups, we proceeded with testing between two groups as suggested by Fisher to protect the least statistically significant difference in multiple testing. A $P$ value of 0.05 was considered statistically significant.

\section{Ethics and trial registration}

The trial was monitored by the regional good clinical practice unit, filed with EudraCT (2016-002237-30, 21.11.2016), approved by The Regional Ethics Committee (S-20160090), and conducted in accordance with the Helsinki Declaration. All participants signed informed written consent. The trial was registered with the Danish Data Protection Agency (16/35979), and data were collected using REDCap 7.0.11 (Vanderbilt University, Nashville, Tennessee, United States). Authorship was appointed in accordance with ICMJE guidelines.

\section{Results}

A total of 1707 patients were screened for eligibility and 517 invitations were sent. We included 180 eligible consecutive patients that responded and fulfilled the criteria. We included $140(78 \%)$ participants at center one, and 40 (22\%) participants at center two (flowchart, Fig.3). Demographics and center-specific randomization for each group are shown in - Table 1. Mean age at inclusion was 59 years (range 32-70) and $52 \%$ were male. Capsule excretion rate, bowel cleansing grade, complete examination rate and capsule transit time of colon in the three groups are shown in - Table 2. Capsule excretion within recording time was achieved in $70 \%(95 \% \mathrm{Cl}$ : $58-80)$ in Group A, $73 \%(95 \% \mathrm{Cl}: 61-83)$ in Group B and in $68 \%(95 \% \mathrm{Cl}: 56-79)$ in Group C. The highest excretion rate was achieved in Group B, but was not statistically significant different from the excretion rates in Group $A$ and $C$. Bowel cleansing grade was statistically significant different in Group B, compared to Group A $(P=0.03)$, but Group $C$ was no different from Group A $(P=0.4)$. Complete examination rate with both capsule excretion and clinically adequate bowel cleansing was $65 \%$ (95\% Cl: $53-77), 72 \%(95 \% \mathrm{Cl}: 61-83)$ and $62 \%(95 \% \mathrm{Cl}$ : $50-74)$ in Group A, B and C respectively, and the differences were not statistically significant. In those who achieved capsule excretion, mean capsule transit time of colon was 252 minutes, 227 minutes and 206 minutes in Group A, B and C respectively, and not statistically significant different. Polyp detection rate was higher in grade 2 to 4 cleansing ( $57 \%-72 \%$ ) compared to 


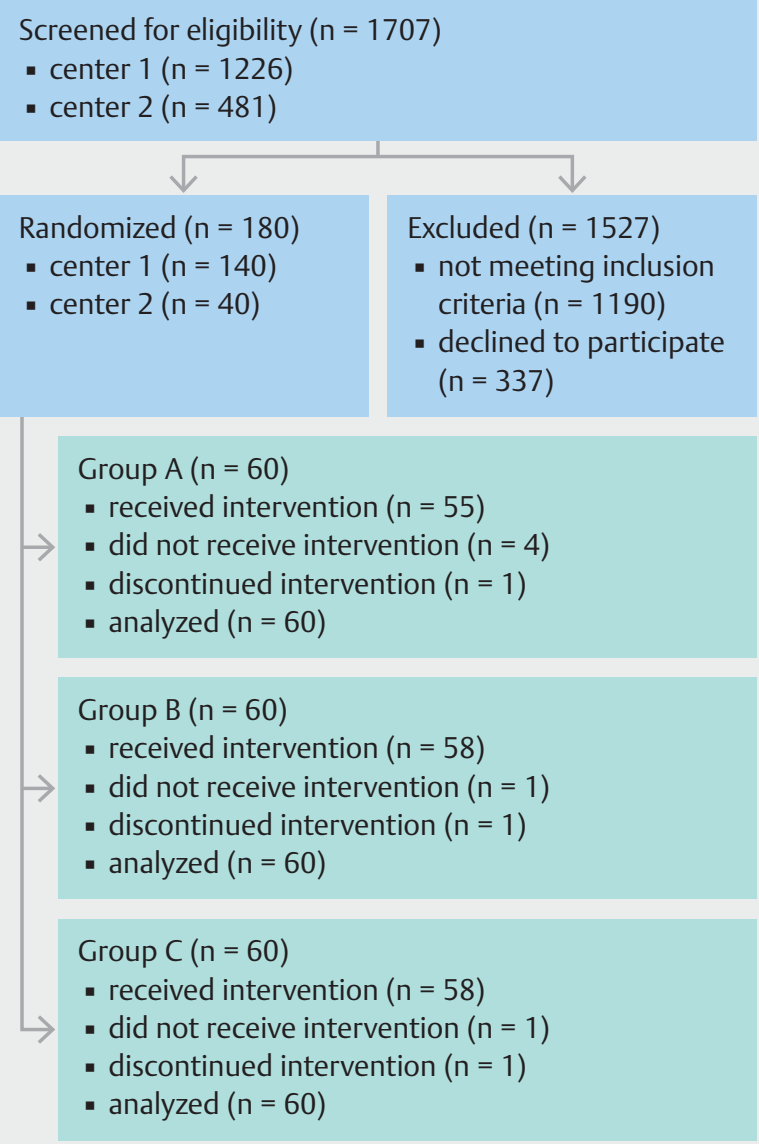

Fig. 3 Study flowchart

grade 1 (41\%) but without statistically significant difference ( $\triangleright$ Table 3 ). Mean number of polyps found was statistically significant higher in grades 2 to $4(1.61-2.06)$ compared to grade 1 (0.47). Overall tolerability was a statistically significant different in Group A compared to Group C $(P=0.02)$. Patient-reported compliance with the booster procedure was good (>75\%) in $82 \%$ (95\% Cl: $72-91), 78 \%$ (95\% Cl: $68-89$ ) and $82 \%(95 \% \mathrm{Cl}$ :
$72-91$ ) in Group A, B and C, respectively, with no statistically significant difference among groups.

\section{Adverse events}

No adverse events (AEs) with the video capsule were seen. A total of 9 participants experienced AEs during the study. Six patients experienced severe vomiting during the preparation with PEG and decided not to continue with the protocol. One patient experienced mild stomach pain and hunger sensation during the preparation with PEG, leading to food intake and termination of the protocol. One participant had a self-limiting rash after ingestion of the booster medication in Group C, but completed the protocol. One participant had sudden onset of vaginal bleeding after ingestion of the booster medication in Group B, leading to admission and observation in the gynecologic department and termination of the protocol.

\section{Discussion}

There were no statistically significant differences in excretion rates and completion rates among the three groups in this trial. Group $B$ had a better bowel cleansing grade compared to Group $A$, and the Group C booster medication was less well tolerated compared to Group A. Given the results with capsule excretion, bowel preparation and patient tolerability, we find that the sulfate-based saline solution booster in Group B performed well. In all three groups, the completion rate was still suboptimal and not comparable to colonoscopy or other published results on colon capsule endoscopy using similar boosters $[3,8,9,11]$. These trials, however, are limited by either small sample sizes (Nastou D et al., Togashi K et al. and Spada C et al.) or lack of intention to treat design with a large number of post hoc exclusions (Rex DK et al.)A reason for the suboptimal outcome might be that the bowel preparation of 2 - $L$ split-dose PEG solution is not as good as 4-L split-dose PEG solution, as reported in a randomized trial [4], leading to poor performance of all three boosters. Another explanation could be the outpatient design of the current study leading to poor patient compliance. This is somewhat contradicted by the self-reported compliance in this study. Estimation of bowel cleansing quality remains a very subjective matter. Although the scale used here has been

- Table 1 Participant demographics, and center-specific stratified randomization.

\begin{tabular}{|c|c|c|c|}
\hline & Group A & Group B & Group C \\
\hline \multicolumn{4}{|l|}{ Sex, N (\%) } \\
\hline - Male & $28(47)$ & $35(58)$ & $30(50)$ \\
\hline - Female & $32(53)$ & $25(42)$ & $30(50)$ \\
\hline \multicolumn{4}{|l|}{ Age, years } \\
\hline - Mean (Range) & $59(34-70)$ & $58(38-70)$ & $58(32-70)$ \\
\hline \multicolumn{4}{|c|}{ Center randomization, N (\%) } \\
\hline - Center 1 & $47(78)$ & $47(78)$ & $46(77)$ \\
\hline - Center 2 & $13(22)$ & $13(22)$ & $14(23)$ \\
\hline
\end{tabular}


$\checkmark$ Table2 Main results.

\begin{tabular}{|c|c|c|c|}
\hline & Group A & Group B & Group C \\
\hline \multicolumn{4}{|l|}{ Capsule excretion } \\
\hline . \% $(95 \% \mathrm{Cl})$ & $70(58-80)$ & $73(61-83)$ & $68(56-79)$ \\
\hline \multicolumn{4}{|c|}{ Colon transit-time, min. } \\
\hline - Mean $(95 \% \mathrm{Cl})$ & $252(209-295)$ & $227(188-266)$ & $206(164-249)$ \\
\hline \multicolumn{4}{|c|}{ Bowel cleansing grade, N (\%) } \\
\hline - Excellent (4) & $6(10)$ & $11(18.3)$ & $11(18)$ \\
\hline - $\operatorname{Good}(3)$ & $26(43)$ & $32(53.3)$ & $24(40)$ \\
\hline - Fair (2) & $15(25)$ & $14(23.3)$ & $18(30)$ \\
\hline - Poor (1) & $13(22)$ & $3(5.1)$ & $7(12)$ \\
\hline \multicolumn{4}{|c|}{ Complete examination } \\
\hline - \% $(95 \% \mathrm{Cl})$ & $65(52-77)$ & $72(61-83)$ & $62(50-74)$ \\
\hline
\end{tabular}

- Table 3 Polyp findings according to bowel cleansing grade.

\begin{tabular}{|l|l|l|l|l|}
\hline & \multicolumn{2}{|c|}{ Bowel cleansing grade } & $\mathbf{3}$ & $\mathbf{4}$ \\
\hline Polyp detection rate, $\%(95 \% \mathrm{Cl})$ & $\mathbf{1}$ & $\mathbf{2}$ & $72(62-82)$ & $57(39-75)$ \\
\hline Mean number of polyps, $\mathrm{N}(95 \% \mathrm{Cl})$ & $41(18-64)$ & $64(50-78)$ & $2.01(1.54-2.48)$ & $1.61(0.82-2.40)$ \\
\hline
\end{tabular}

validated with good inter-observer variability, it has not been thoroughly investigated as to how it translates into clinical practice. Our results suggest that grade 2 (fair) has comparable polyp findings to the better grades. Bowel cleansing grade should probably be seen in conjunction with the indication for CCE before judging whether it is clinically adequate or not. In theory, numerous conditions and medications can affect gastrointestinal motility and CCE performance, but no such research identifying these factors exists for CCE. The randomized design was chosen to overcome these issues but unidentified confounding factors could be unaccounted for in this study.

\section{Conclusion}

Further studies are needed comparing the efficiency and tolerability of both bowel preparation and booster medication in colon capsule endoscopy in randomized, intention-to-treat designs.

\section{Acknowledgements}

The study was supported by the Danish Cancer Society, The Research Foundation of the Health Care Region of Southern Denmark and Odense University Hospital Research Grant. Medtronic provided a total of 180 free PillCam Colon 2 devices for the study. The authors thank data manager Jakob Uffelmann, Sundhed.dk and statistician René dePont Christensen of the Research Unit for General Practice, Odense University Hospital.

\section{Competing interests}

This trial and several other trials performed by this research group have received funding from Medtronic.

References

[1] Kobaek-Larsen M, Kroijer R, Dyrvig AK et al. Back-to-back colon capsule endoscopy and optical colonoscopy in colorectal cancer screening individuals. Colorectal Dis 2018; 20: 479-485

[2] Spada C, Pasha SF, Gross SA et al. Accuracy of First- and Second-Generation Colon Capsules in Endoscopic Detection of Colorectal Polyps: A Systematic Review and Meta-analysis. Clin Gastroenterol Hepatol 2016; 14: $1533-1543$

[3] Rex DK, Adler SN, Aisenberg J et al. Accuracy of capsule colonoscopy in detecting colorectal polyps in a screening population. Gastroenterology 2015; 148: $948-957$

[4] Arguelles-Arias F, San-Juan-Acosta M, Belda A et al. Preparations for colon capsule endoscopy. Prospective and randomized comparative study between two preparations for colon capsule endoscopy: PEG 2 liters + ascorbic acid versus PEG 4 liters. Rev Esp Enferm Dig 2014; 106: $312-317$

[5] Saito Y, Saito S, Oka S et al. Evaluation of the clinical efficacy of colon capsule endoscopy in the detection of lesions of the colon: prospective, multicenter, open study. Gastrointest Endosc 2015; 82: 861 869

[6] Adler SN, Hassan C, Metzger Y et al. Second-generation colon capsule endoscopy is feasible in the out-of-clinic setting. Surg Endosc 2014; 28: $570-575$ 
[7] Holleran G, Leen R, O'Morain C et al. Colon capsule endoscopy as possible filter test for colonoscopy selection in a screening population with positive fecal immunology. Endoscopy 2014; 46: 473-478

[8] Spada C, Hassan C, Barbaro B et al. Colon capsule versus CT colonography in patients with incomplete colonoscopy: a prospective, comparative trial. Gut 2015; 64: 272-281

[9] Nastou D, Palmer H, Lewandowski JL et al. Sa1099 Colon capsule transit times and image quality in patients prepared with polyethylene glycol+ascorbate $(\mathrm{PEG}+\mathrm{A})$ and booster of either $\mathrm{PEG}+\mathrm{A}$ or gastrografin and low dose sodium phosphate (NaP). Gastroenterology148: 223

[10] Spada C, Hassan C, Munoz-Navas M et al. Second-generation colon capsule endoscopy compared with colonoscopy. Gastrointest Endosc 2011; 74: $581-589$

[11] Togashi K, Fujita T, Utano K et al. Gastrografin as an alternative booster to sodium phosphate in colon capsule endoscopy: safety and efficacy pilot study. Endosc Int Open 2015; 3: 659-661

[12] Hagel AF, Gabele E, Raithel M et al. Colon capsule endoscopy: detection of colonic polyps compared with conventional colonoscopy and visualization of extracolonic pathologies. Can J Gastroenterol Hepatol 2014; 28: 77-82

[13] Farnbacher M], Krause HH, Hagel AF et al. QuickView video preview software of colon capsule endoscopy: reliability in presenting colorectal polyps as compared to normal mode reading. Scand J Gastroenterol 2014; 49: 339-346
[14] Adrian-de-Ganzo Z, Alarcon-Fernandez O, Ramos L et al. Uptake of colon capsule endoscopy vs colonoscopy for screening relatives of patients with colorectal cancer. clin gastroenterol Hepatol 2015; 13 : $2293-2301$

[15] Eliakim R, Yassin K, Niv Y et al. Prospective multicenter performance evaluation of the second-generation colon capsule compared with colonoscopy. Endoscopy 2009; 41: 1026-1031

[16] Negreanu L, Babiuc R, Bengus A et al. PillCam Colon 2 capsule in patients unable or unwilling to undergo colonoscopy. World J Gastrointest Endosc 2013; 5: 559-567

[17] Rondonotti E, Borghi C, Mandelli G et al. Accuracy of capsule colonoscopy and computed tomographic colonography in individuals with positive results from the fecal occult blood test. Clin Gastroenterol Hepatol 2014; 12: $1303-1310$

[18] Morgan DR, Malik PR, Romeo DP et al. Initial US evaluation of secondgeneration capsule colonoscopy for detecting colon polyps. BMJ open gastroenterol 2016: 3

[19] Pandis N, Chung B, Scherer RW et al. CONSORT 2010 statement: extension checklist for reporting within person randomised trials. BM] 2017; 357: j2835

[20] Leighton JA, Rex DK. A grading scale to evaluate colon cleansing for the PillCam COLON capsule: a reliability study. Endoscopy 2011; 43: $123-127$ 